\title{
Laser Review
}

\section{Mesospheric Na Wind/Temperature Lidar}

\author{
Chester S. GARDNER* ${ }^{*}$ and George C. PAPEN*
}

(Received October 10, 1994)

\begin{abstract}
Meteoric ablation in the upper atmosphere is the source of a dense layer of atomic Na between $80-105 \mathrm{~km}$ with maximum densities averaging $4 \times 10^{3} \mathrm{~cm}^{-3}$. The Na layer is a sensitive tracer of the wind, thermal, and density structure of this region and can be probed with exceptional accuracy and resolution using resonance fluorescence lidar techniques. Modern narrowband tunable lasers are now being used in $\mathrm{Na}$ lidar systems to measure routinely winds and temperatures with accuracies approaching $\pm 1 \mathrm{~m} / \mathrm{s}$ and $\pm 1 \mathrm{~K}$. We describe the $\mathrm{Na}$ Wind/ Temperature lidar technique and present examples of observations made during the recent ALOHA-93 Campaign in Hawaii.
\end{abstract}

Key Words: Lidar, Na, Winds, Temperature, Mesosphere

\section{Introduction}

Until recently, in situ rocket probes and powerful mesosphere-stratosphere-troposphere radars were the predominant techniques used to explore middle atmosphere composition and structure. However, during the past decade, numerous lidar techniques have been developed to probe this region with superb accuracy and resolution. Ozone, Rayleigh, aerosol, and resonance fluorescence lidars are now playing central roles in many middle atmosphere studies.

It has long been recognized that the $\mathrm{Na}$ layer is a sensitive tracer of the density, temperature, and wind structure between 80 and $105 \mathrm{~km}$. Although the first lidar observations of $\mathrm{Na}$ density profiles were made in the 1960 s, soon after the invention of the tunable dye laser, it was not until the mid-1980s that the linewidth and frequency stability of dye lasers improved sufficiently for reliable temperature observations to be conducted routinely ${ }^{1,2)}$. Further improvements in laser technology now make $\mathrm{Na}$ Doppler wind measurements possible.

Temperature and wind observations can be obtained by probing the fine structure of the $\mathrm{Na} \mathrm{D}_{2}$ fluorescence spectrum with a tunable narrow-band pulsed laser. For accurate temperature measurements $( \pm 2 \mathrm{~K})$ the required absolute tuning accuracy and frequency stability of the laser is $20 \mathrm{MHz} \mathrm{rms}$ or less. An order of magnitude improvement in absolute tuning precision and stability $(\sim 2 \mathrm{MHz})$ is required for accurate radial wind measurements $( \pm 1$ $\mathrm{m} / \mathrm{s})$. These performance requirements can be achieved using state-of-the-art $\mathrm{cw}$ ring laser and pulsed amplifier technologies. Several groups have recently demonstrated $\mathrm{Na}$ wind and temperature measurement using this approach ${ }^{3,4)}$. In this paper we describe the measurement technique and present examples of mesospheric wind and temperature profiles acquired with the University of Illinois $\mathrm{Na}$ lidar system during the ALOHA-93 (1993 Airborne Lidar and Observations of the Hawaiian Airglow) Campaign.

\section{Na Wind/Temperature Lidar Technique}

The Na $D_{2}$ absorption spectrum consists of six hyperfine resonance lines that are broadened predominantly by inhomogeneous thermal processes. This spectrum is further broadened by the laser lineshape when probed in a lidar experiment. The broadened absorption spectrum is a doublet with the frequency of the major $\mathbf{D}_{2 a}$ peak located approximately $1.77 \mathrm{GHz}$ below the $D_{2 b}$ peak (Fig. 1a). The thermally broadened width of each doublet is approximately $1.1 \mathrm{GHz}$ full width at half maximum (FWHM). The ratio of the absorption spectrum at

* Department of Electrical and Computer Engineering, University of Illinois (Urbana-Champaign Urbana, IL 61801 USA) 


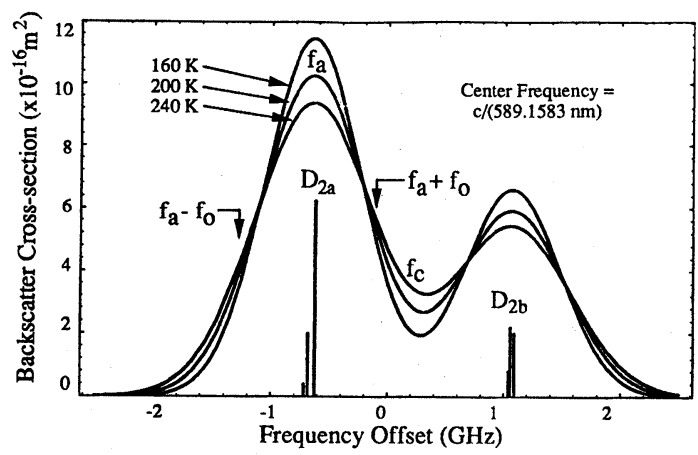

(a)

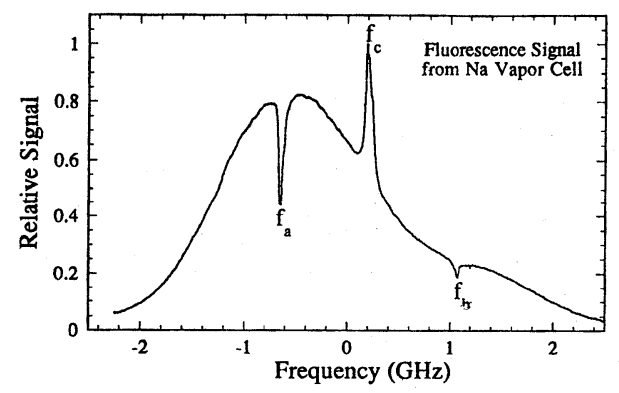

(b)

Fig. 1 (a) $\mathrm{Na} \mathrm{D}_{2}$ thermally broadened absorption spectrum plotted as a function of frequency for three temperatures. The $D_{2 a}$ and $D_{2 b}$ hyperfine groups and the four laser frequencies used in the ALOHA-93 experiment are depicted as well. (b) Measured $\mathrm{Na}$ vapor fluorescence spectrum. The Doppler-free features at $f_{a}$ and $f_{c}$ are prominently displayed.

the minimum between the $D_{2 a}$ and $D_{2 b}$ peaks to the spectrum at the $D_{2 a}$ peak is a sensitive function of temperature. Similarly, the ratio of the spectrum on either side of the $D_{2 a}$ peak is a sensitive function of the Doppler shift. Temperature is sensed by tuning a narrowband pulsed laser alternately to the $D_{2 a}$ peak frequency $f_{a}$ and to the crossover resonancc frequency $f_{c}$, which is near the minimum between the $D_{2 a}$ and $D_{2 b}$ peaks (Fig. 1), and recording the backscattered fluorescence signal from the Na layer at each frequency. The Doppler shift and hence radial winds are sensed by tuning the laser alternately to two different frequencies in the wings of the $D_{2 a}$ absorption peak, viz., at $f_{a} \pm f_{o}$ where $f_{o}=600 \mathrm{MHz}$. Detailed theoretical discussions of the temperature and wind measurement techniques can be found in Bills et al. ${ }^{5)}$ and Papen et al. ${ }^{6)}$.

The narrowband laser used in the ALOHA-93 ex- periment included a cw ring dye laser oscillator and three-stage pulsed dye amplifier with an average output power of $1 \mathrm{~W}$ at 30 pps. The cw laser oscillator was tuned precisely by observing the Dopplerfree structure of the $\mathrm{Na} D_{2}$ fluorescence spectrum in a vapor cell (Fig. 1b) and by monitoring the laser oscillator signal using a temperature-stabilized confocal etalon. The lidar was installed at the Air Force Maui Optical Site near the summit of Haleakala at an altitude of $3055 \mathrm{~m}$ and interfaced with the $0.8 \mathrm{~m}$ diameter Beam Director Telescope which provided full scanning capabilities. Vertical and horizontal winds were measured by pointing at zenith and $15^{\circ}$ off zenith to the north and east. The power aperture product of the lidar system was $0.5 \mathrm{Wm}^{2}$.

Unlike conventional radar, the Doppler shift associated with fluorescence absorption is $-V_{R} / \lambda$, where $V_{\mathrm{R}}$ is the radial wind velocity toward the laser and $\lambda=589.158 \mathrm{~nm}$ is the center wavelength of the $D_{2}$ emission. The minus sign appears because the $\mathrm{Na}$ atoms are excited by a laser pulse that has been Doppler shifted to a higher frequency (assuming $V_{R}>0$ ), which is equivalent to shifting the absorption spectrum to a lower frequency. The backscattered signal results from spontaneous emission of the excited $\mathrm{Na}$ atoms. Because the fluorescence signal is proportional to the absorption cross section and is detected by a broadband receiver, only the Doppler shift associated with the absorption process is observed. When the lidar is pointed to the east, the Doppler shift $\left(f_{d}\right)$ is

$$
f_{d}=-V_{\mathrm{R}} / \lambda=(u / \lambda) \sin 15^{\circ}+(w / \lambda) \cos 15^{\circ}
$$

where $u$ is the zonal wind (eastward winds are positive) and $w$ is the vertical wind (upward winds are positive). The shift is $\sim 1.7 \mathrm{MHz} /(\mathrm{m} / \mathrm{s})$.

The primary sources of error are photon noise and laser frequency errors. By approximating the $\mathrm{Na} \mathrm{D}_{2}$ emission spectrum as the sum of two Gaussian distributions centered at the $D_{2 a}$ and $D_{2 b}$ frequencies, approximate expressions for the errors can be derived. The photon noise errors for radial velocity $\left(V_{\mathrm{R}}\right)$ and temperature $(T)$ are Bills fu al., 5); Papen fu al., ${ }^{6)}$

$$
\begin{aligned}
& \Delta V_{\mathrm{R}}(\mathrm{m} / \mathrm{s}) \simeq \frac{490 \mathrm{~m} / \mathrm{s}}{\sqrt{N_{s}\left(f_{a}, z\right)}} \\
& \Delta T(\mathrm{~K}) \simeq \frac{510 \mathrm{~K}}{\sqrt{N_{s}\left(f_{a}, z\right)}}
\end{aligned}
$$

$N_{s}\left(f_{a}, z\right)$ is the equivalent photon count in the range bin centered at altitude $z$, assuming the laser was tuned to frequency $f_{a}^{-}$during the time period that profiles were actually collected at 
the four frequencies $f_{a}-f_{o}, f_{a}, f_{a}+f_{o}$, and $f_{c} . N_{s}$ is given by the lidar equation and for typical system parameters can be written approximately as

$$
\begin{aligned}
N_{s}\left(f_{a}, z\right)= & 40 P(\mathrm{~W}) A\left(\mathrm{~m}^{2}\right) \\
& \cdot n_{s}\left(\mathrm{~cm}^{-3}\right) \Delta t(\min ) \Delta z(\mathrm{~km})
\end{aligned}
$$

where $P$ is the average output power of the laser, $A$ is the aperture area of the receiving telescope, $n_{s}$ is the $\mathrm{Na}$ density at altitude $z, \Delta t$ is the time required to obtain the profiles at the four different frequencies, and $\Delta z$ is the altitude resolution of the wind or temperature measurement.

At the peak of the Na layer near $92 \mathrm{~km}$ altitude the density averages approximately $4000 \mathrm{~cm}^{-3}$ and for ALOHA-93 the power-aperture product of the lidar was $0.5 \mathrm{Wm}^{2}$. If the integration period is $6 \mathrm{~min}$ and the vertical resolution is $0.5 \mathrm{~km}$, then $N_{s}\left(f_{a}, 92\right.$ km) $\mathrm{O} \simeq 2.4 \times 10^{5}$ and the approximate radial velocity and temperature errors are respectively, $1 \mathrm{~m} / \mathrm{s}$ and $1 \mathrm{~K}$.

The radial wind and temperature errors contributed by laser frequency errors are given by

$$
\begin{aligned}
& \Delta V_{\mathrm{R}}(\mathrm{m} / \mathrm{s}) \simeq 0.4 \Delta f(\mathrm{MHz}) \\
& \Delta T(\mathrm{~K}) \simeq 0.1 \Delta f(\mathrm{MHz}) .
\end{aligned}
$$

By using the Doppler free features in the $\mathrm{Na}$ cell fluorescence spectrum as frequency markers (Figure 1b) and modern ring-dye laser oscillators as we did for ALOHA-93, an absolute tuning accuracy of 2 $\mathrm{MHz}$ or better can be achieved. Consequently, the radial velocity and temperature errors caused by errors in laser tuning can be held to better than 1 $\mathrm{m} / \mathrm{s}$ and $0.25 \mathrm{~K}$.

\section{Observations During the ALOHA-93 Cam- paign}

To illustrate the capabilities of Na W/T lidars, contour plots of the $\mathrm{Na}$ density and temperature measured on 6 October 1993 during ALOHA-93 are presented in Fig. 2. Contour plots of the vertical and zonal winds measured on this same night are presented in Fig. 3. These data are interesting because a major sporadic $\mathrm{Na}$ layer formed near $92 \mathrm{~km}$ at approximately 0900 UT and then moved downward throughout the remainder of the observation period with an apparent velocity of approximately $15 \mathrm{~cm} / \mathrm{s}$. The temperature in the vacinity of the sporadic $\mathrm{Na}$ layer was considerably warmer than the surrounding region, reaching values higher than $240 \mathrm{~K}$. The vertical wind velocity exhibited no unusual characteristics near the sporadic layer. The vertical velocity fluctuations throughout the height range of observations were approximately $\pm 5 \mathrm{~m} / \mathrm{s}$. In con- trast the zonal winds varied between -80 and 100 $\mathrm{m} \mathrm{s}$ with a strong shear in the region of the sporadic $\mathrm{Na}$ layer. This is illustrated more clearly in Fig. 4 where the $\mathrm{Na}$ density, temperature, zonal wind, and vertical shear of zonal winds at $0905 \mathrm{UT}$ are plotted versus altitude. The maximum shear occurred at the peak of the sporadic Na layer at $92 \mathrm{~km}$. Throughout the sporadic layer the temperature was approximately $40 \mathrm{~K}$ warmer than the surrounding regions.

The chemical and dynamic processes responsible for the formation of sporadic $\mathrm{Na}$ layers are not yet well understood. However, data such as these tually understanding this puzzling phenomenon. These data also illustrate the potential of $\mathrm{Na} \mathrm{W} / \mathrm{T}$

(a) Na Density $\left(\times 1000 \mathrm{~cm}^{-3}\right)$

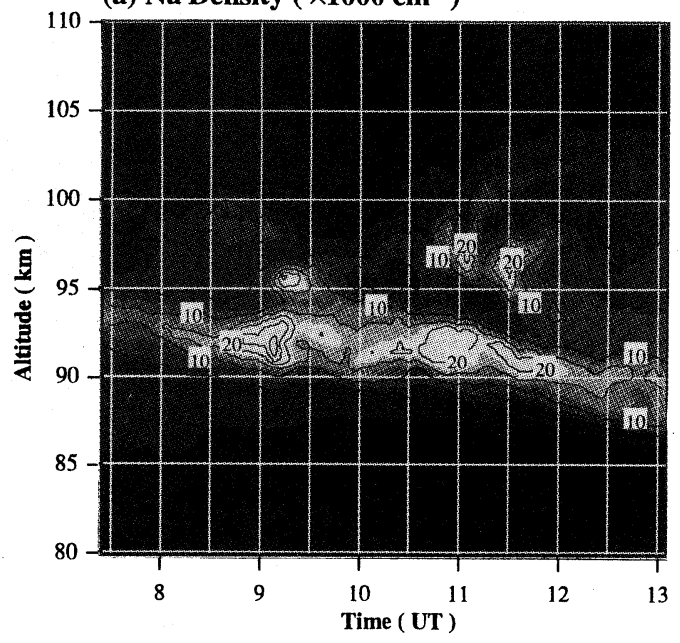

(b) Temperature ( $\mathrm{K}$ )

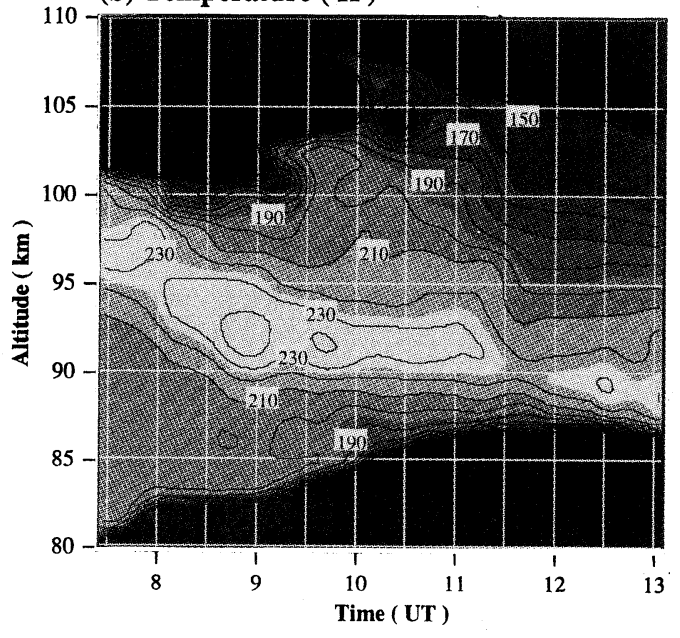

Fig. 2 Contour plots of the (a) Na density and (b) atmospheric temperature measured on 6 October 1993 at Haleakala, Maui during ALOHA-93. 
(a) Vertical Wind ( $\mathrm{m} / \mathrm{sec}$ )

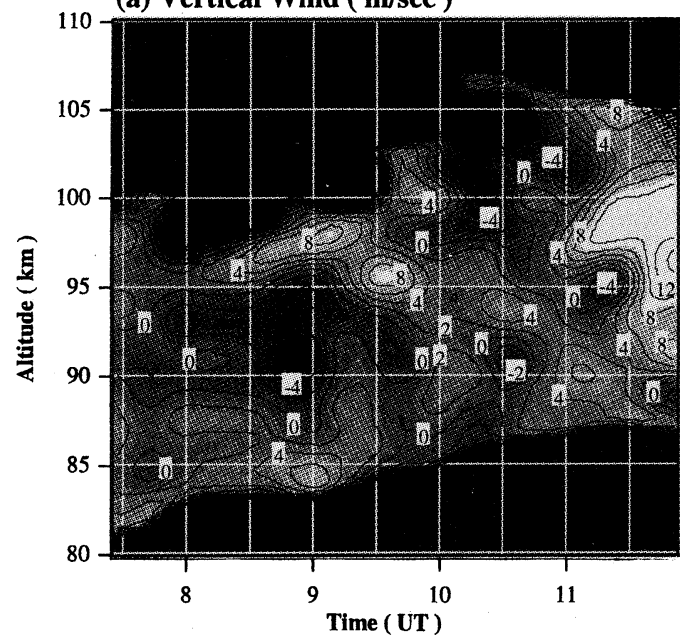

(b) Zonal Wind ( $\mathrm{m} / \mathrm{sec}$ )

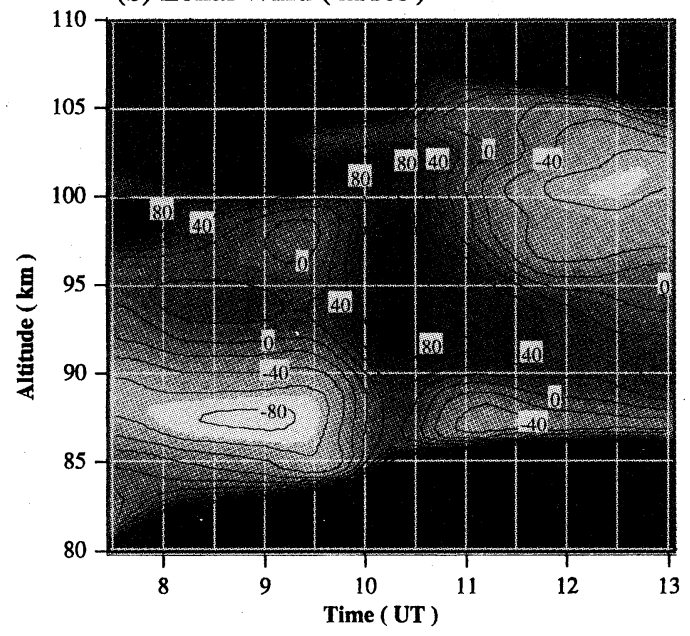

Fig. 3 Contour plots of the (a) vertical and (b) zonal winds measured on 6 October 1993 at Haleakala, Maui.

lidars in mesospheric studies. The performance of existing systems is essentially photon noise limited. (a) $\mathrm{Na}\left(\times 1000 \mathrm{~cm}^{-3}\right)$

(b) $\mathbf{u}(\mathrm{m} / \mathrm{sec})$
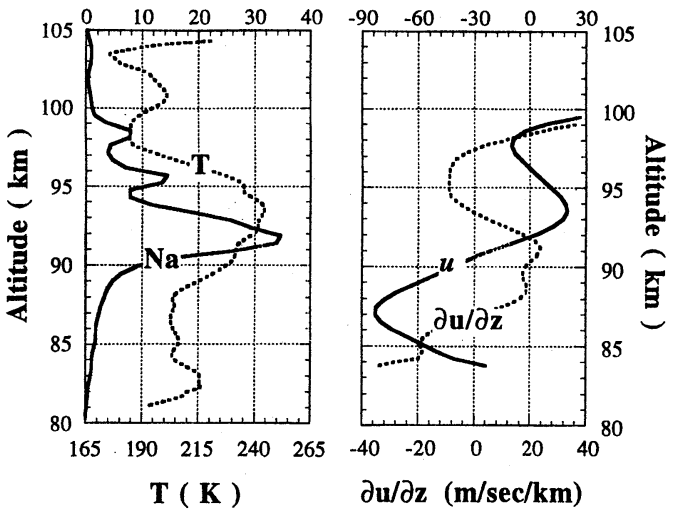

Fig. 4 (a) Na density and temperature profiles measured at 905 UT on 6 October 1993. (b) Zonal wind profile and the vertical shear of the zonal wind profile measured at 905 UT on 6 October 1993.

Improvements in measurement accuracy and resolution can only be obtained by increasing the power aperture product of the lidar. By using $3 \mathrm{~m}$ class astronomical telescopes, improvements of 15 to 20 over the ALOHA-93 lidar are easily achievable, thus yielding measurements with significantly improved spatial and temporal resolution.

\section{References}

1) R. P. Neuber, vonder Gathen and U. von Zahn: J. Geophys. Res. 93 (1988) 11093.

2) C. Y. She, R. E. Bills, H. Latifi, J. R. Yu, R. J. Alvarez II and C. S. Gardner: Geophys. Res. Lett. 17 (1990) 929.

3) R. E. Bills, C. S. Gardner and S. F. Franke: J. Geophys. Res. 96 (1991) 22701.

4) C. Y. She and J. R. Yu: Geophy. Res. Lett. 21 (1994) 1771.

5) R. E. Bills, C. S. Gardner and C. Y. She: Opt. Eng. 30 (1991) 13.

6) G. C. Papen, W. M. Pfenninger and D. M. Simmich: Appl. Opt., in press (1994). 